\section{A new pathway to pathology?}

It's not every day that a new anatomical pathway is discovered, so the recent finding that the primate thalamus receives dopaminergic inputs from various brain regions was bound to be greeted with interest. This robust and complex dopaminergic system could explain thalamic abnormalities that have been reported in conditions such as schizophrenia and Parkinson's disease.

Despite the fact that dopamine was detected in the thalamus of primates more than 20 years ago, the idea that this structure receives a significant dopamine innervation has not been acknowledged, perhaps because the rodent thalamus appears to receive only scant dopaminergic inputs.

Sánchez-González and colleagues looked for dopaminergic axons in the thalamic nuclei of adult humans and macaque monkeys using antibodies against tyrosine hydroxylase, dopamine and the dopamine transporter, DAT. They found that dopaminergic axons profusely target the thalamus, and that this innervation is particularly prominent in specific association, limbic and motor thalamic nuclei. Interestingly, the DAT content of dopaminergic axons varies across the thalamus, which suggests that dopaminergic signalling is more

spatially and temporally restricted in some thalamic regions than in others.

Using retrograde tract tracing combined with immunohistochemistry, the researchers showed that dopamine innervation of the thalamus originates bilaterally in several dopaminergic neuronal populations, including those of the hypothalamus, the periaqueductal grey and the ventral mesencephalon.

The thalamus relays information to the neocortex from subcortical centres and from other cortical areas. This relay function is modulated such that the flow of information reflects the wakefulness of the animal and current attentional and behavioural demands. The finding of a widespread thalamic dopaminergic system leads to the inevitable conclusion that dopamine can modulate thalamic information transfer according to a range of environmental and internal signals. A closer inspection of this system could further our understanding of the dopamine-related disorders schizophrenia and Parkinson's disease.

\section{Rebecca Craven}

\section{(1) References and links} ORIGINAL RESEARCH PAPER SánchezGonzález, M. A. et al. The primate thalamus is a key target for brain dopamine. J. Neurosci. 25, 6076-6083 (2005)

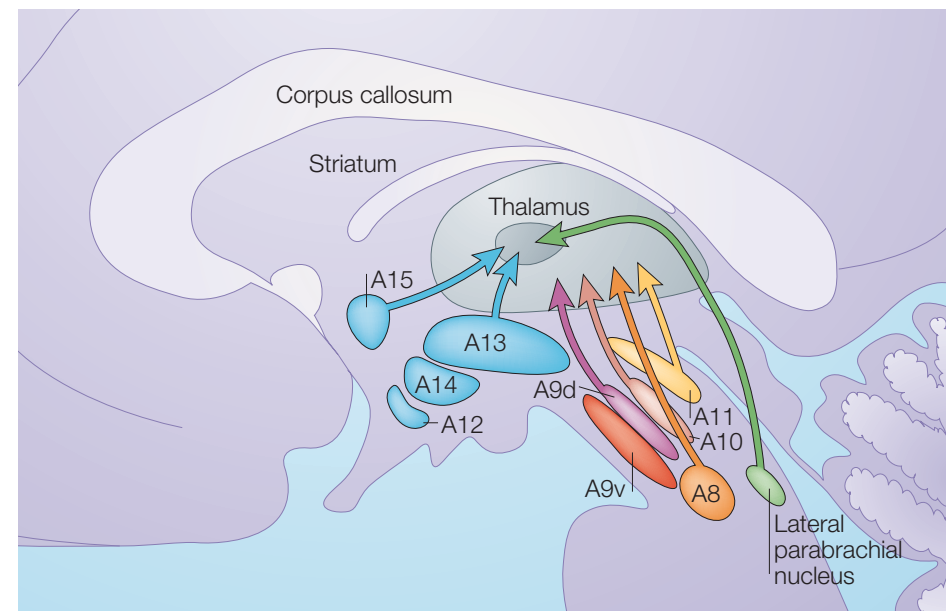

Dopaminergic projections to the thalamus. Inputs from groups A8-A11 express the dopamine transporter, DAT, whereas those from groups A12-A15 and the lateral parabrachial nucleus express little or no DAT. Image courtesy of C. Cavada, Departamento de Anatomía, Histología y Neurociencia, Universidad Autonoma de Madrid, Spain.
IN BRIEF

\section{NEUROTRANSMISSION}

Crystal structure of a bacterial homologue of $\mathrm{Na}^{+} / \mathrm{Cl}^{-}$-dependent neurotransmitter transporters.

Yamashita, A. et al. Nature 24 July 2005 (10.1038/nature03978)

$\mathrm{Na}^{+} / \mathrm{Cl}^{-}$-dependent transporters mediate the uptake of neurotransmitters from the synaptic cleft into neurons and glia. The crystal structure of a bacterial homologue of these transporters shows how the transporter binds its substrate, leucine, and two $\mathrm{Na}^{+}$ions within the protein core. The structure also defines the internal and external gates of the transporter and sheds light on how its substrate and ion selectivity are determined.

\section{TASTE}

\section{Positive selection on a high-sensitivity allele of the} human bitter-taste receptor TAS2R16.

Soranzo, N. et al. Curr. Biol. 15, 1257-1265 (2005)

Sequencing of the human bitter taste receptor (a G-proteincoupled receptor encoded by TAS2R16) from different populations shows several signatures of positive selection, and a common ancestor of the derived N172 variant that is estimated to have existed during the middle Pleistocene, before early humans spread out from Africa. The ability to identify bitter toxins might have been evolutionarily crucial as humans expanded into different environments, and this could have driven positive selection of this receptor.

\section{NEURAL CODING}

Independent codes for spatial and episodic memory in hippocampal neuronal ensembles.

Leutgeb, S. et al. Science 309, 619-623 (2005)

Recordings of hippocampal neurons in rats placed in either identical recording chambers in different locations, or different recording chambers in the same location, show two independent coding schemes. When cues varied but location did not, the firing rates of cells differed but their spatial selectivity remained the same. When location rather than cues changed, both the firing rates and spatial selectivity of the neurons varied. These independent coding schemes might allow the hippocampus to encode both spatial and episodic memories.

\section{NEUROGENESIS}

\section{Retrospective birth dating of cells in humans.}

Spalding, K. L. et al. Cell 122, 133-143 (2005)

The levels of the isotope ${ }^{14} \mathrm{C}$ in our atmosphere rose steeply during the testing of nuclear weapons and then decreased exponentially after 1963 . This variation can be used to establish the age of individual cells, because the level of ${ }^{14} \mathrm{C}$ in genomic DNA closely parallels that of the atmosphere. Spalding et al. describe this technique and use it to date the generation of cells in the adult human cortex. As expected, non-neuronal cells were generated more recently than cortical neurons, which were as old as the individual. 